CUBO A Mathematical Journal

Vol.12, № 03, (241-253). October 2010

\title{
On the Weyl Transform with Symbol in the Gel'fand-Shilov Space and its Dual Space
}

\author{
YASUYUKI OKA \\ Department of Mathematics, Sophia University \\ 7-1 Kioicho, Chiyoda-ku, Tokyo 102-8554, Japan \\ email: yasuyu-o@hoffman.cc.sophia.ac.jp
}

\begin{abstract}
In this paper, we claim two subjects. One is that the Weyl transform with symbol in the Gel'fand-Shilov space $\mathscr{S}_{r}^{r}, r \geq \frac{1}{2}$, is a trace class operator. The other one is that the Weyl transform with symbol in the generalized function $\left(\mathscr{S}_{r}^{r}\right)^{\prime}, r \geq \frac{1}{2}$, is a continuous linear transformation from the Gel'fand-Shilov space $\mathscr{S}_{r}^{r}$ to $\left(\mathscr{S}_{r}^{r}\right)^{\prime}$. As $r>1$, Z. LozanovCrvenković and D. Perišić have proved in [6] this result. Our second claim includes their result.
\end{abstract}

\section{RESUMEN}

En este artículo afirmamos dos asuntos. El primero es que la transformada de Weyl con símbolo en el espacio de Gel'fand-Shilov $\mathscr{S}_{r}^{r}, r \geq \frac{1}{2}$, es un operador de clase trazo. El segundo asunto es que la transformación de Weyl con símbolo en las funciones generalizadas $\left(\mathscr{S}_{r}^{r}\right)^{\prime}, r \geq \frac{1}{2}$, es una transformación lineal continua del espacio Gel'fand-Shilov $\mathscr{S}_{r}^{r}$ to $\left(\mathscr{S}_{r}^{r}\right)^{\prime}$. Como $r>1$, Z. Lozanov-Crvenković y D. Perišić probaron en [6] este resultado. Nuestro resultado incluye su resultado.

Key words and phrases: Weyl transform, Gel'fand-Shilov space, Fourier-Wigner transform, trace class operator, Schwartz's kernel theorem.

Math. Subj. Class.: 46F05; 46F15; 81R15; 81S40. 


\section{Introduction}

The subject of this article is to show the properties, as operators, of the Weyl transform with the symbol in the Gel'fand-Shilov space $\mathscr{S}_{r}^{r}, r \geq 1 / 2$, and its dual space $\left(\mathscr{S}_{r}^{r}\right)^{\prime}, r \geq 1 / 2$.

The Weyl transform was first considered by Hermann Weyl arising in quantum mechanics in [14] and the properties of the Weyl transform as operators have been studied by many mathematicians. See for instance, [1], [6], [9], [11], [12], [13], [15] and others. These investigations are mainly to consider the correspondence between the functional space, in which the symbol belongs, and the operator class, in which the Weyl transform belongs.

There exist two remarkable results about these considerations: first, that the Weyl transform with the symbol in Schwartz class is a trace class operator in [13]; and secondly, that the Weyl transform with the symbol in $\left(\mathscr{S}_{r}^{r}\right)^{\prime}, r>1$, is a continuous and linear maps from $\mathscr{S}_{r}^{r}\left(\mathbb{R}^{d}\right)$ to $\left(\mathscr{S}_{r}^{r}\right)^{\prime}\left(\mathbb{R}^{d}\right)$ in [6]. Our discussion is principally aimed at slightly developing these two results. They depend on two areas of study: first, the correspondence between the Weyl transform with the symbol in $\mathscr{S}_{r}^{r}, r \geq 1 / 2$, and the sequence space with some exponential decrease, and secondly, the study of the Schwartz's kernel theorem for $\left(\mathscr{S}_{r}^{r}\right)^{\prime}, r \geq 1 / 2$. We consider these subjects in detail.

The plan of the paper is as follows. In the next section we introduce some properties of the Gel'fand-Shilov space. In section 3 we treat the Weyl transform with symbol in $\mathscr{S}_{r}^{r}$. In section 4 we show the Schwartz's kernel theorem for $\left(\mathscr{S}_{r}^{r}\right)^{\prime}$ and the property of the Weyl transform with symbol in generalized functions. Through this article we always treat the index $r \geq 1 / 2$.

\section{The Gel'fand-Shilov Space $\mathscr{S}_{r}^{r}$ and its Dual $\left(\mathscr{S}_{r}^{r}\right)^{\prime}$}

First of all, we give some notations. We use a multi-index $\alpha \in \mathbb{Z}_{+}^{d}$, namely, $\alpha=\left(\alpha_{1} \cdots \alpha_{d}\right)$, where $\alpha_{i} \in \mathbb{Z}$ and $\alpha_{i} \geq 0$. So, for $x \in \mathbb{R}^{d}, x^{\alpha}=x_{1}^{\alpha_{1}} \cdots x_{d}^{\alpha_{d}}$ and $\partial_{x}^{\alpha}=\partial_{x_{1}}^{\alpha_{1}} \cdots \partial_{x_{d}}^{\alpha_{d}}$, where $\partial_{x_{j}}^{\alpha_{j}}=\left(\frac{\partial}{\partial x_{j}}\right)^{\alpha_{j}}$.

Definition 1 ([4]). Let $A, B \in(0, \infty)^{d}$. For $r=\left(r_{1}, \cdots, r_{d}\right)$ and $r_{i} \geq 0,1 \leq i \leq d$, $\mathscr{S}_{r, A}^{r, B}\left(\mathbb{R}^{d}\right)=\left\{\varphi \in C^{\infty}\left(\mathbb{R}^{d}\right) \mid \forall \delta \in(0, \infty)^{d}, \forall \rho \in(0, \infty)^{d}, \exists C_{\delta \rho} \geq 0\right.$ s.t.

where

$$
\left.\left|x^{k} \partial_{x}^{q} \varphi(x)\right| \leq C_{\delta \rho}(A+\delta)^{k}(B+\rho)^{q} k^{k r} q^{q r}, \forall k, q \in \mathbb{Z}_{+}^{d}\right\},
$$

$$
\begin{aligned}
& (A+\delta)^{k}=\left(A_{1}+\delta_{1}\right)^{k_{1}} \cdots\left(A_{d}+\delta_{d}\right)^{k_{d}}, \\
& (B+\rho)^{q}=\left(B_{1}+\rho_{1}\right)^{q_{1}} \cdots\left(B_{d}+\rho_{d}\right)^{q_{d}} .
\end{aligned}
$$


The space $\mathscr{S}_{r, A}^{r, B}\left(\mathbb{R}^{d}\right)$ is a Fréchet space with the semi-norms

$$
\|\varphi\|^{\delta \rho}=\sup _{x, k, q} \frac{\left|x^{k} \partial_{x}^{q} \varphi(x)\right|}{(A+\delta)^{k}(B+\rho)^{q} k^{k r} q^{q r}}, \delta_{i}, \rho_{i}=1, \frac{1}{2}, \frac{1}{3} \cdots .
$$

The space $\mathscr{S}_{r}^{r}\left(\mathbb{R}^{d}\right)$ is given by the inductive limit

$$
\mathscr{S}_{r}^{r}\left(\mathbb{R}^{d}\right)=\lim _{A, B \rightarrow \infty} \mathscr{S}_{r, A}^{r, B}\left(\mathbb{R}^{d}\right)
$$

The Gel'fand-Shilov space is the subspace of the Schwartz class $\mathscr{S}\left(\mathbb{R}^{d}\right)$.

Let $a \in(0, \infty)^{d}$ be $a=\frac{r}{e A^{\frac{1}{r}}}$. For any $a, B \in(0, \infty)^{d}$, we define the space $\mathscr{S}_{r, a}^{r, B}\left(\mathbb{R}^{d}\right)$ by $\mathscr{S}_{r, a}^{r, B}\left(\mathbb{R}^{d}\right)=\left\{\varphi \in C^{\infty}\left(\mathbb{R}^{d}\right) \mid \forall \delta, \rho \in(0, \infty)^{d}, \exists C_{\delta \rho}>0\right.$ s.t. $\left|\partial_{x}^{q} \varphi(x)\right| \leq C_{\delta \rho}(B+\rho)^{q} q^{q r} e^{-a_{\delta}|x|^{\frac{1}{r}}}$, $\left.\forall k, q \in \mathbb{Z}_{+}^{d}\right\}$ where $a_{\delta}=\frac{r}{e(A+\delta)^{\frac{1}{r}}}$ and

$$
\|\varphi\|_{\delta \rho}=\sup _{x, \beta} \frac{\left|\partial_{x}^{\beta} \varphi(x)\right|}{(B+\rho)^{\beta} \beta^{\beta r} e^{-a_{\delta}|x|^{\frac{1}{r}}}} .
$$

The Gel'fand-Shilov spaces $\mathscr{S}_{r}^{r}\left(\mathbb{R}^{d}\right)$ enjoy the following properties [4]:

Proposition 1. Let $\left\{\varphi_{j}\right\}$ be a sequence in $\mathscr{S}_{r}^{r}\left(\mathbb{R}^{d}\right)$. Then we obtain

$$
\varphi_{j} \longrightarrow 0 \text { as } j \longrightarrow+\infty \text { in } \mathscr{S}_{r}^{r}
$$

if and only if there are positive constants $B$ and a such that

$$
\sup _{x, \beta} \frac{\left|\partial_{x}^{\beta} \varphi_{j}(x)\right|}{B^{\beta} \beta^{\beta r} e^{-a|x|^{\frac{1}{r}}}} \longrightarrow 0 \text { as } j \longrightarrow+\infty .
$$

Proposition 2. $\quad$ (i) $\mathscr{S}_{r}^{r} \equiv\{0\}, 0<r<\frac{1}{2}$.

(ii) For $r_{1}<r_{2}, \mathscr{S}_{r_{1}}^{r_{1}}$ is included in $\mathscr{S}_{r_{2}}^{r_{2}}$ and $\mathscr{S}_{r_{1}}^{r_{1}}$ is dense in $\mathscr{S}_{r_{2}}^{r_{2}}$.

(iii) Let $\hat{\mathscr{S}}_{r}^{r}$ be the image of the Fourier transform of $\mathscr{S}_{r}^{r}$. Then $\hat{\mathscr{S}}_{r}^{r}=\mathscr{S}_{r}^{r}$.

Remark 1. As $r=1$, the Gel'fand-Shilov space $\mathscr{S}_{1}^{1}\left(\mathbb{R}^{d}\right)$ is known to be isomorphism to the space of test functions of the Fourier-hyperfunctions [7].

We define the Hermite functions $\left\{h_{n}(x)\right\}_{n=0,1,2 \cdots}$ on $\mathbb{R}^{1}$ by

$$
h_{n}(x)=\left(2^{n} n !\right)^{-\frac{1}{2}} \pi^{-\frac{1}{4}}(-1)^{n} e^{\frac{x^{2}}{2}}\left(\frac{d}{d x}\right)^{n} e^{-x^{2}} .
$$


It is known that the set $\left\{h_{n}(x)\right\}_{n=0,1,2, \cdots}$ is a complete orthonormal system in $L^{2}\left(\mathbb{R}^{1}\right)$. That is, for any $f$ in $L^{2}\left(\mathbb{R}^{1}\right)$,

$$
f(x)=\sum_{n=0}^{\infty} a_{n} h_{n}(x) \text { in } L^{2}\left(\mathbb{R}^{1}\right)
$$

where $a_{n}=\left(f, h_{n}\right)=\int_{\mathbb{R}^{1}} f(x) h_{n}(x) d x$. This expansion is called the Hermite expansions and $\left\{a_{n}\right\}_{n=0,1,2 \ldots}$ is called the Hermite coefficients. For $d$-dimensions, the Hermite functions on $\mathbb{R}^{d}$ is defined by

$$
h_{\alpha}(x)=h_{\alpha_{1}}\left(x_{1}\right) \otimes \cdots \otimes h_{\alpha_{d}}\left(x_{d}\right), \quad \alpha \in \mathbb{Z}_{+}^{d}, x \in \mathbb{R}^{d} .
$$

The set $\left\{h_{\alpha}(x)\right\}_{\alpha \in \mathbb{Z}_{+}^{d}}$ is also a complete orthonormal system in $L^{2}\left(\mathbb{R}^{d}\right)$.

Proposition 3 ([17]). Let $\phi \in \mathscr{S}_{r}^{r}\left(\mathbb{R}^{d}\right), r \geq \frac{1}{2}$. Then there exist some constants $C>0$ and $L \in$ $(0, \infty)^{d}$ such that

$$
\phi=\sum_{|\alpha|=0}^{\infty}\left(\phi, h_{\alpha}\right) h_{\alpha} \text { and }\left|\left(\phi, h_{\alpha}\right)\right| \leq C \exp \left(-L \alpha^{\frac{1}{2 r}}\right)
$$

Conversely, if $\left|a_{\alpha}\right| \leq C \exp \left(-L \alpha^{\frac{1}{2 r}}\right)$ for some constants $C>0$ and $L \in(0, \infty)^{d}$, then the series $\sum_{|\alpha|=0}^{\infty} a_{\alpha} h_{\alpha}(x)$ converges to a function in $\mathscr{S}_{r}^{r}\left(\mathbb{R}^{d}\right)$, where $h_{\alpha}(x)$ is the Hermite function.

Definition 2. We denote by $\left(\mathscr{S}_{r}^{r}\right)^{\prime}\left(\mathbb{R}^{d}\right)$ the dual space of the Gel'fand-Shilov space $\mathscr{S}_{r}^{r}\left(\mathbb{R}^{d}\right)$.

\section{The Weyl Transform with Symbol in $\mathscr{S}_{r}^{r}$}

As quantization from classical mechanics to quantum mechanics, $\mathrm{H}$. Weyl introduced the operator $\mathbb{W}(F)$ as follows: for any $F \in \mathscr{S}\left(\mathbb{R}^{2 d}\right)$,

$$
\mathscr{W}(F) \varphi(\xi)=\iint_{\mathbb{R}^{2 d}} F(x, y)[\pi(x, y) \varphi](\xi) d x d y, \varphi \in L^{2}\left(\mathbb{R}^{d}\right),
$$

where $[\pi(x, y) \varphi](\xi)=e^{i\left(x \cdot \xi+\frac{1}{2} x \cdot y\right)} \varphi(\xi+y)$. We call this transform $\mathscr{W}(F)$ the Weyl transform with symbol $F$. The Weyl transform $\mathscr{W}(F)$ is also expressed by the following matrix element: for any $\varphi, \psi \in L^{2}\left(\mathbb{R}^{d}\right)$,

$$
\begin{aligned}
(\mathscr{W}(F) \varphi, \psi) & =\iint_{\mathbb{R}^{2 d}} F(x, y)(\pi(x, y) \varphi, \psi) d x d y \\
& =\iint_{\mathbb{R}^{2 d}} F(x, y) V(\varphi, \psi)(x, y) d x d y,
\end{aligned}
$$

where $V(\varphi, \psi)(x, y)$ is the Fourier-Wigner transform of $\varphi$ and $\psi$ defined by

$$
V(\varphi, \psi)(x, y)=(2 \pi)^{-\frac{d}{2}} \int_{\mathbb{R}^{d}} e^{i x \cdot p} \varphi\left(p+\frac{y}{2} \overline{\psi\left(p-\frac{y}{2}\right)} d p .\right.
$$


The Fourier-Wigner transform has the following property, see for example [5]. To be definite, we shall repeat here the proof.

Proposition 4. Let $\varphi, \psi \in \mathscr{S}_{r}^{r}\left(\mathbb{R}^{d}\right), r \geq \frac{1}{2}$. Then $V(\varphi, \psi) \in \mathscr{S}_{r}^{r}\left(\mathbb{R}^{2 d}\right)$.

Proof. It follows from Proposition 2 (iii) that a partial Fourier transform of the first variables is a continuous map from $\mathscr{S}_{r}^{r}$ to $\mathscr{S}_{r}^{r}$, so it suffices to show that if $\varphi, \psi$ are in $\mathscr{S}_{r}^{r}\left(\mathbb{R}^{d}\right)$, then $\varphi\left(p+\frac{y}{2}\right) \bar{\psi}\left(p-\frac{y}{2}\right)$ is in $\mathscr{S}_{r}^{r}\left(\mathbb{R}^{2 d}\right)$. Suppose $\varphi, \psi \in \mathscr{S}_{r}^{r}\left(\mathbb{R}^{d}\right)$. Since

$$
p^{\alpha}=\sum_{|k|=0}^{\alpha}\left(\begin{array}{l}
\alpha \\
k
\end{array}\right)\left(p+\frac{y}{2}\right)^{k}\left(p-\frac{y}{2}\right)^{\alpha-k} \text { and } y^{\beta}=\sum_{|l|=0}^{\beta}\left(\begin{array}{l}
\beta \\
l
\end{array}\right)\left(p+\frac{y}{2}\right)^{l}(-1)^{|\beta-l|}\left(p-\frac{y}{2}\right)^{\beta-l},
$$

we have that

$$
\begin{aligned}
p^{\alpha} y^{\beta} \partial_{p}^{\gamma} \partial_{y}^{\delta} \varphi\left(p+\frac{y}{2}\right) \bar{\psi}\left(p-\frac{y}{2}\right)= & \sum_{k, l, m, n}^{\alpha, \beta, \gamma, \delta}\left(\begin{array}{l}
\alpha \\
k
\end{array}\right)\left(\begin{array}{l}
\beta \\
l
\end{array}\right)\left(\begin{array}{l}
\gamma \\
m
\end{array}\right)\left(\begin{array}{l}
\delta \\
n
\end{array}\right)(-1)^{|\beta-l|}\left(p+\frac{y}{2}\right)^{k+l}\left(p-\frac{y}{2}\right)^{\alpha+\beta-k-l} \\
& \times \partial_{p}^{m} \partial_{y}^{n} \varphi\left(p+\frac{y}{2}\right) \partial_{p}^{\gamma-m} \partial_{y}^{\delta-n} \bar{\psi}\left(p-\frac{y}{2}\right)
\end{aligned}
$$

Set $u=p+\frac{y}{2}$ and $v=p-\frac{y}{2}$, then

$$
(3.2)=\sum_{k, l, m, n}^{\alpha, \beta, \gamma, \delta}\left(\begin{array}{l}
\alpha \\
k
\end{array}\right)\left(\begin{array}{l}
\beta \\
l
\end{array}\right)\left(\begin{array}{l}
\gamma \\
m
\end{array}\right)\left(\begin{array}{l}
\delta \\
n
\end{array}\right)(-1)^{|\beta+\delta-l-n|}\left(\frac{1}{2}\right)^{|\delta|} u^{k+l} v^{\alpha+\beta-k-l} \partial_{u}^{m+n} \partial_{v}^{\gamma-m+\delta-n} \varphi(u) \bar{\psi}(v) .
$$

So we obtain that for any $\alpha, \beta \gamma, \delta \in \mathbb{Z}_{+}^{d}$,

$$
\begin{aligned}
\left|p^{\alpha} y^{\beta} \partial_{p}^{\gamma} \partial_{y}^{\delta} \varphi\left(p+\frac{y}{2}\right) \bar{\psi}\left(p-\frac{y}{2}\right)\right| \leq & \sum_{k, l, m, n}^{\alpha, \beta, \gamma, \delta}\left(\begin{array}{l}
\alpha \\
k
\end{array}\right)\left(\begin{array}{l}
\beta \\
l
\end{array}\right)\left(\begin{array}{l}
\gamma \\
m
\end{array}\right)\left(\begin{array}{l}
\delta \\
n
\end{array}\right)\left|u^{k+l} \partial_{u}^{m+n} \varphi(u)\right|\left|v^{\alpha+\beta-k-l} \partial_{v}^{\gamma-m+\delta-n} \bar{\psi}(v)\right| \\
\leq & C_{1} C_{2} \sum_{k, l, m, n}^{\alpha, \beta, \gamma, \delta}\left(\begin{array}{l}
\alpha \\
k
\end{array}\right)\left(\begin{array}{l}
\beta \\
l
\end{array}\right)\left(\begin{array}{l}
\gamma \\
m
\end{array}\right)\left(\begin{array}{l}
\delta \\
n
\end{array}\right) A_{1}^{k+l} A_{2}^{\alpha+\beta-k-l} B_{1}^{m+n} B_{2}^{\gamma-m+\delta-n} \\
\times & (k+l)^{(k+l) r}(\alpha+\beta-k-l)^{(\alpha+\beta-k-l) r}(m+n)^{(m+n) r} \\
& (\gamma-m+\delta-n)^{(\gamma-m+\delta-n) r}
\end{aligned}
$$

for suitable constants $A_{1}, A_{2}, B_{1}, B_{2} \in(0, \infty)^{d}$ and $C_{1}, C_{2}>0$. Thus we have that

$$
(3.3) \leq C_{3} A_{3}^{\alpha+\beta} B_{3}^{\gamma+\delta}(\alpha+\beta)^{(\alpha+\beta) r}(\gamma+\delta)^{(\gamma+\delta) r}
$$

for some constants $A_{3}, B_{3} \in(0, \infty)^{d}$ and $C_{3}>0$. Since

$$
(\alpha+\beta)^{(\alpha+\beta) r} \leq e^{\alpha r} e^{\beta r} \alpha^{\alpha r} \beta^{\beta r} \text { and }(\gamma+\delta)^{(\gamma+\delta) r} \leq e^{\gamma r} e^{\delta r} \gamma^{\gamma r} \delta^{\delta r},
$$


if we put $A_{4}=A_{3} e^{r}$ and $B_{4}=B_{3} e^{r}$, then we have

$$
\text { (3.4) } \leq C_{3} A_{4}^{\alpha+\beta} B_{4}^{\gamma+\delta} \alpha^{\alpha r} \beta^{\beta r} \gamma^{\gamma r} \delta^{\delta r} .
$$

Hence we obtain that there exist constants $A_{4}, B_{4} \in(0, \infty)^{d}$ and $C_{3}>0$ such that

$$
\left|p^{\alpha} y^{\beta} \partial_{p}^{\gamma} \partial_{y}^{\delta} \varphi\left(p+\frac{y}{2}\right) \bar{\psi}\left(p-\frac{y}{2}\right)\right| \leq C_{3} A_{4}^{\alpha+\beta} B_{4}^{\gamma+\delta} \alpha^{\alpha r} \beta^{\beta r} \gamma^{\gamma r} \delta^{\delta r}
$$

for any $\alpha, \beta, \gamma$ and $\delta \in \mathbb{Z}_{+}^{d}$. This completes the proof of Proposition 4.

A straightforward computation with (3.1) shows that if $F(x, y) \in \mathscr{S}\left(\mathbb{R}^{2 d}\right)$, then we have

$$
\mathscr{W}(F) \varphi(p)=\int_{\mathbb{R}^{d}} K\left(p, p^{\prime}\right) \varphi\left(p^{\prime}\right) d p^{\prime}, \varphi \in L^{2}\left(\mathbb{R}^{d}\right),
$$

where the kernel $K\left(p, p^{\prime}\right)=\mathscr{F}_{1}^{-1} F\left(\frac{p+p^{\prime}}{2}, p^{\prime}-p\right)$. Here $\mathscr{F}_{1}^{-1} F$ denotes the inverse Fourier transform of $F$ in the first variables.

The Weyl transform has the following fundamental properties, see for example [15].

Proposition 5. (i) If the symbol $F$ is in $L^{1}\left(\mathbb{R}^{2 d}\right)$, then the Weyl transform $\mathbb{W}(F)$ is a bounded operator on $L^{2}\left(\mathbb{R}^{d}\right)$,

(ii) Let the symbol $F$ be in $L^{2}\left(\mathbb{R}^{2 d}\right)$. Then the Weyl transform $\mathscr{W}(F)$ is the Hilbert Schmidt operator on $L^{2}\left(\mathbb{R}^{d}\right)$. Conversely let $\Phi$ be the Hilbert-Schmidt operator. Then there exists $F \in L^{2}\left(\mathbb{R}^{d}\right)$ such that

$$
\Phi=\mathscr{W}(F)
$$

We obtain the following result concerning on the property of the Weyl transform $\mathscr{W}(F)$ with the symbol $F$ in $\mathscr{S}_{r}^{r}\left(\mathbb{R}^{d}\right), r \geq 1 / 2$ :

Theorem 1. Let $\mathbb{W}\left(\mathscr{S}_{r}^{r}\left(\mathbb{R}^{2 d}\right)\right)$ be the set of all the Weyl transforms with the symbol in the Gel'fand-Shilov space $\mathscr{S}_{r}^{r}\left(\mathbb{R}^{2 d}\right)$. Then

$\mathscr{W}\left(\mathscr{S}_{r}^{r}\left(\mathbb{R}^{2 d}\right)\right)=\left\{R \in \mathscr{B}\left(L^{2}\left(\mathbb{R}^{d}\right)\right) \mid \exists a, a^{\prime} \in(0, \infty)^{d}, \exists C>0\right.$ such that $\left.\left|\left(R h_{\alpha}, h_{\beta}\right)\right| \leq C e^{-a|\alpha| \frac{1}{2 r}} e^{-a^{\prime}|\beta| \frac{1}{2 r}}, \forall \alpha, \beta \in \mathbb{Z}_{+}^{d}\right\}$,

where $\mathscr{B}\left(L^{2}\left(\mathbb{R}^{d}\right)\right)$ is the set of all bounded operators on $L^{2}\left(\mathbb{R}^{d}\right)$ and $h_{\alpha}, h_{\beta}$ are the Hermite functions.

Proof. Let $\mathscr{G}=\left\{R \in \mathscr{B}\left(L^{2}\left(\mathbb{R}^{d}\right)\right) \mid \exists a, a^{\prime} \in(0, \infty)^{d}, \exists C>0\right.$ such that $\left|\left(R h_{\alpha}, h_{\beta}\right)\right| \leq C e^{-a|\alpha|^{\frac{1}{2 r}}}$ $\left.e^{-a^{\prime}|\beta| \frac{1}{2 r}}, \forall \alpha, \beta \in \mathbb{Z}_{+}^{d}\right\}$. By (3.5) and Proposition 2 (iii), it is apparent that the symbol $F \in$ 
$\mathscr{S}_{r}^{r}\left(\mathbb{R}^{2 d}\right)$ if and only if the kernel $K \in \mathscr{S}_{r}^{r}\left(\mathbb{R}^{2 d}\right)$. By proposition 3 and Fubini's theorem, we have that

$$
\begin{aligned}
\left|\left(\mathscr{W}(F) h_{\alpha}, h_{\beta}\right)\right| & \leq\left|\left(\int_{\mathbb{R}^{d}} K\left(p, p^{\prime}\right) h_{\alpha}\left(p^{\prime}\right) d p^{\prime}, h_{\beta}(p)\right)\right| \\
& =\left|\iint_{\mathbb{R}^{2} d} K\left(p, p^{\prime}\right) h_{\alpha}\left(p^{\prime}\right) h_{\beta}(p) d p^{\prime} d p\right| \\
& =\left|\left(K, h_{\alpha} \otimes h_{\beta}\right)\right| \\
& \leq C e^{-a|\alpha| \frac{1}{2 r}} e^{-a^{\prime}|\beta| \frac{1}{2 r}}
\end{aligned}
$$

for some constants $a, a^{\prime} \in(0, \infty)^{d}$ and $C>0$. Therefore $\mathscr{W}(F) \in \mathscr{G}$. Conversely, let $R_{1} \in \mathscr{G}$. Then

$$
\begin{aligned}
\sum_{|\alpha|=0}^{\infty}\left\|R_{1} h_{\alpha}\right\|_{L^{2}\left(\mathbb{R}^{d}\right)}^{2} & =\sum_{|\alpha|=0}^{\infty}\left(R_{1} h_{\alpha}, R_{1} h_{\alpha}\right) \\
& \leq \sum_{|\alpha|=0|\beta|=0}^{\infty} \sum_{|\alpha|=0|\beta|=0}^{\infty}\left|\left(R_{1} h_{\alpha}, h_{\beta}\right) \|\left(h_{\beta}, R_{1} h_{\alpha}\right)\right| \\
& =\sum_{|\alpha|}^{\infty} \sum^{-2 a|\alpha|^{\frac{1}{2 r}}} e^{-2 a^{\prime}|\beta|^{\frac{1}{2 r}}}<+\infty .
\end{aligned}
$$

Hence $R_{1}$ is the Hilbert-Schmidt operator. Therefore it follows from Proposition 5 that there exists $G \in L^{2}\left(\mathbb{R}^{2 d}\right)$ such that $\mathscr{W}(G)=R_{1}$. Then from (3.5) there exists $C>0$ and $a, a^{\prime} \in$ $(0, \infty)^{d}$ such that

$$
\left|\left(R_{1} h_{\alpha}, h_{\beta}\right)\right|=\left|\left(\mathscr{W}(G) h_{\alpha}, h_{\beta}\right)\right|=\left|\left(K, h_{\alpha} \otimes h_{\beta}\right)\right| \leq C e^{-a|\alpha|^{\frac{1}{2 r}}} e^{-a^{\prime}|\beta|^{\frac{1}{2 r}}} .
$$

By proposition 3, we obtain that $K \in \mathscr{S}_{r}^{r}\left(\mathbb{R}^{2 d}\right)$ and so is $G$.

Corollary 1. If $F, G \in \mathscr{S}_{r}^{r}\left(\mathbb{R}^{2 d}\right)$, then there exists $H \in \mathscr{S}_{r}^{r}\left(\mathbb{R}^{2 d}\right)$ such that $\mathscr{W}(H)=\mathscr{W}(F) \mathscr{W}(G)$

Proof. Let $F, G \in \mathscr{S}_{r}^{r}\left(\mathbb{R}^{2 d}\right)$. Then we have that

$$
\begin{aligned}
\left|\left(\mathscr{W}(F) \mathscr{W}(G) h_{\alpha}, h_{\beta}\right)\right| & =\left|\left(\mathbb{W}(G) h_{\alpha}, \mathscr{W}(F)^{*} h_{\beta}\right)\right| \\
& \leq \sum_{\gamma}\left|\left(\mathbb{W}(G) h_{\alpha}, h_{\gamma}\right)\right|\left|\left(h_{\gamma}, \mathscr{W}(F)^{*} h_{\beta}\right)\right| \\
& =\sum_{\gamma}\left|\left(\mathscr{W}(G) h_{\alpha}, h_{\gamma}\right)\right|\left|\left(\mathscr{W}(F) h_{\gamma}, h_{\beta}\right)\right| \\
& \leq C e^{-a|\alpha|^{\frac{1}{2 r}}} e^{-b|\beta|^{\frac{1}{2 r}}} \sum_{\gamma} e^{-\left(a^{\prime}+b^{\prime}\right)|\gamma| \frac{1}{2 r}} \\
& =C^{\prime} e^{-a|\alpha|^{\frac{1}{2 r}}} e^{-b|\beta| \frac{1}{2 r}}
\end{aligned}
$$


for suitable constants $a, a^{\prime}, b, b^{\prime} \in(0, \infty)^{d}$ and $C, C^{\prime}>0$. Hence we obtain that $\mathscr{W}(F) \mathscr{W}(G) \in$ $\mathscr{G}$. Therefore it follows from Theorem 1 that $H$ is in $\mathscr{S}_{r}^{r}\left(\mathbb{R}^{2 d}\right)$ such that $\mathscr{W}(H)=\mathscr{W}(F) \mathscr{W}(G)$.

Remark 2. It is known that $\mathscr{W}(F) \mathscr{W}(G)=\mathscr{W}\left(F *_{\frac{1}{4}} G\right)$, where

$$
\left(F *{ }_{\frac{1}{4}} G\right)(x, y)=\iint_{\mathbb{R}^{2 d}} F(x-\xi, y-\eta) G(\xi, \eta) e^{\frac{i}{4} \times 2(y \cdot \xi-x \cdot \eta)} d \xi d \eta .
$$

So if $F, G \in \mathscr{S}_{r}^{r}\left(\mathbb{R}^{2 d}\right)$, then $\left(F *{ }_{\frac{1}{4}} G\right) \in \mathscr{S}_{r}^{r}\left(\mathbb{R}^{2 d}\right)$ from Corollary 1 .

Definition 3. We denote by $S_{1}$ the family of all trace class operators defined as follows: for $A \in \mathscr{B}\left(L^{2}\left(\mathbb{R}^{d}\right)\right)$, there exists an orthonormal basis $\left\{v_{k}\right\}$ of $L^{2}\left(\mathbb{R}^{d}\right)$ such that

$$
\sum_{k}\left\|A v_{k}\right\|_{L^{2}\left(\mathbb{R}^{d}\right)}<\infty
$$

Proposition 6. Let $A \in \mathscr{B}\left(L^{2}\left(\mathbb{R}^{d}\right)\right)$. If $\left\{v_{j}\right\}$ is an orthonormal basis of $L^{2}\left(\mathbb{R}^{d}\right)$ then

$$
\sum_{j}\left\|A v_{j}\right\|_{L^{2}\left(\mathbb{R}^{d}\right)} \leq \sum_{j, k}\left|\left(A v_{j}, v_{k}\right)\right| .
$$

Proof. It is obvious as $A v_{j}=0$, so it suffices to show as $A v_{j} \neq 0$. Let $w_{j}$ be an unit vector for any index $j$. Then we have

$$
\begin{aligned}
\sum_{j}\left|\left(A^{*} w_{j}, v_{j}\right)\right| & =\sum_{j}\left|\left(w_{j}, A v_{j}\right)\right| \\
& =\sum_{j}\left|\sum_{k}\left(w_{j}, v_{k}\right)\left(v_{k}, A v_{j}\right)\right| \\
& =\sum_{j}\left|\sum_{k}\left(A^{*} v_{k}, v_{j}\right)\left(w_{j}, v_{k}\right)\right| \\
& \leq \sum_{j, k}\left|\left(A^{*} v_{k}, v_{j}\right)\right| \\
& =\sum_{j, k}\left|\left(A v_{j}, v_{k}\right)\right| .
\end{aligned}
$$

Choose $w_{j}=\frac{A v_{j}}{\left\|A v_{j}\right\|}$. The inequality now follows. Indeed from (3.6) we have

$\sum_{j}\left|\left(w_{j}, A v_{j}\right)\right|=\sum_{j}\left|\left(\frac{A v_{j}}{\left\|A v_{j}\right\|}, A v_{j}\right)\right|=\sum_{j} \frac{1}{\left\|A v_{j}\right\|}\left|\left(A v_{j}, A v_{j}\right)\right|^{2}=\sum_{j}\left\|A v_{j}\right\| \leq \sum_{j, k}\left|\left(A v_{j}, v_{k}\right)\right|$.

We obtain the following result from Theorem 1 and Proposition 6:

Corollary 2. The Weyl transform $\mathscr{W}(F)$ with symbol in $\mathscr{S}_{r}^{r}\left(\mathbb{R}^{2 d}\right)$ is of the trace class $S_{1}$.

Remark 3. Since the Gel'fand-Shilov classes are included in the Schwartz class, the preceding Corollary 2 can be seen also as a consequence of the results of A. Voros [13], proving that the Weyl transforms with symbol in the Schwartz class are trace operators. 


\section{On the Weyl Transform with Symbol in $\left(\mathscr{S}_{r}^{r}\right)^{\prime}$}

We first show the Schwartz's kernel theorem for $\left(\mathscr{S}_{r}^{r}\right)^{\prime}, r \geq \frac{1}{2}$, and give the property of the Weyl transform with the symbol in $\left(\mathscr{S}_{r}^{r}\right)^{\prime}, r \geq \frac{1}{2}$, as a corollary of the Schwartz's kernel theorem. S. -Y. Chung, D. Kim and E. G. Lee proved the Schwartz kernel Theorem for $\left(\mathscr{S}_{1}^{1}\right)^{\prime}$ in [2] and Z. Lozanov-Crvenković and D. Perišić gave the Schwartz kernel theorem for $\left(\mathscr{S}_{r}^{r}\right)^{\prime}$ as $r>1$ in [6]. Our result includes their results.

We prove the following Schwartz's kernel theorem for $\left(\mathscr{S}_{r}^{r}\right)^{\prime}, r \geq \frac{1}{2}$, along the idea in [2]:

Theorem 2. Let $k$ be a continuous and linear operator from $\mathscr{S}_{r}^{r}\left(\mathbb{R}_{x_{2}}^{d_{2}}\right)$ to $\left(\mathscr{S}_{r}^{r}\right)^{\prime}\left(\mathbb{R}_{x_{1}}^{d_{1}}\right), r \geq \frac{1}{2}$. Then there exists $K$ in $\left(\mathscr{S}_{r}^{r}\right)^{\prime}\left(\mathbb{R}_{x_{1}}^{d_{1}} \times \mathbb{R}_{x_{2}}^{d_{2}}\right), r \geq \frac{1}{2}$, such that

$$
\langle k \psi, \varphi\rangle=\langle K, \varphi \otimes \psi\rangle,
$$

where $\varphi$ is in $\mathscr{S}_{r}^{r}\left(\mathbb{R}_{x_{1}}^{d_{1}}\right), r \geq \frac{1}{2}$, and $\psi$ is in $\mathscr{S}_{r}^{r}\left(\mathbb{R}_{x_{2}}^{d_{2}}\right), r \geq \frac{1}{2}$.

To prove the Theorem 2, we begin from some preparations. We define the heat kernel $E(x, t)$ by

$$
E(x, t)=\left(\frac{1}{\sqrt{4 \pi t}}\right)^{d} e^{-\frac{|x|^{2}}{4 t}},(x, t) \in \mathbb{R}^{d} \times(0, \infty) .
$$

The heat kernel enjoys the following properties:

$\cdot E(x, t) \in \mathscr{S}\left(\mathbb{R}_{x}^{d}\right)$,

$\cdot \int_{\mathbb{R}^{d}} E(x, t) d x=1$

$\cdot\left(\frac{\partial}{\partial t}-\Delta\right) E(x, t)=0$, in $\mathbb{R}^{d} \times(0, \infty)$.

Moreover we obtain the following estimate on the heat kernel $E(x, t)$ :

Proposition 7 ([16]). For any $\alpha \in \mathbb{Z}_{+}^{d}$, we have

$$
\left|\partial_{x}^{\alpha} E(x, t)\right| \leq E(x, t)(\alpha !)^{\frac{1}{2}}(2 t)^{-|\alpha|}(1+|x|)^{\alpha}, x \in \mathbb{R}^{d}, 0<t \leq \frac{1}{2} .
$$

From this estimate, we immediately obtain the following properties:

Proposition 8. $E(x, t) \in \mathscr{S}_{r}^{r}\left(\mathbb{R}_{x}^{d}\right), r \geq \frac{1}{2}$.

Proposition 9. Let $E(x, t)$ is in $\mathscr{S}_{r, a}^{r, B}$ for any $a, B>0$. Then for every $T>0$ and $\varepsilon>0$, there is a constant $C>0$ such that

$$
\|E(x-\cdot, t)\|_{\delta \rho} \leq C \exp \left[\varepsilon\left(|x|^{\frac{1}{r}}+(1 / t)^{1 /(2 r-1)}\right)\right], x \in \mathbb{R}^{d}, 0<t<T .\left(r>\frac{1}{2}\right)
$$


In the case where $r=1 / 2$, we have the following inequality:

$$
\|E(x-\cdot, t)\|_{\delta \rho} \leq C_{\varepsilon, t} e^{\varepsilon|x|^{2}}, x \in \mathbb{R}^{d}, t>0,\left(r=\frac{1}{2}\right) .
$$

Moreover we need the several propositions, which are the result of C. Dong and T. Matsuzawa [3], to prove Theorem 2 as follows:

Proposition 10 ([3]). Let $\varphi(x) \in \mathscr{S}_{r, a}^{r, B}\left(\mathbb{R}^{d}\right), r \geq 1 / 2$. Then

$$
U(x, t) \equiv \int_{\mathbb{R}^{d}} E(x-y, t) \varphi(y) d y \in \mathscr{S}_{r, a}^{r, B}\left(\mathbb{R}^{d}\right), t>0
$$

and

$$
U(x, t) \rightarrow \varphi(x) \text { in } \in \mathscr{S}_{r, a}^{r, B}\left(\mathbb{R}^{d}\right) \text { as } t \rightarrow 0 .
$$

Proposition 11 ([3]). If Every $C^{\infty}$-function $U(x, t)$ defined in $\mathbb{R}_{+}^{d+1}=\left\{(x, t) \mid x \in \mathbb{R}^{d}, t>0\right\}$ satisfies the conditions:

$$
\left(\frac{\partial}{\partial t}-\Delta\right) U(x, t)=0, \text { in } \mathbb{R}_{+}^{d+1},
$$

and for every $T>0$ and $\varepsilon>0$, there is a constant $C>0$ such that

$$
|U(x, t)| \leq C \exp \left[\varepsilon\left(|x|^{\frac{1}{r}}+(1 / t)^{1 /(2 r-1)}\right)\right], x \in \mathbb{R}^{d}, 0<t<T .\left(r>\frac{1}{2}\right)
$$

In the case where $r=1 / 2, U(x, t)$ has the following inequality:

$$
|U(x, t)| \leq C_{\varepsilon, t} e^{\varepsilon|x|^{2}}, x \in \mathbb{R}^{d}, t>0 .\left(r=\frac{1}{2}\right)
$$

Then $U(x, t)$ can be expressed in the form $U(x, t)=\left\langle u_{y}, E(x-y, t)\right\rangle$ with unique element $u \in$ $\left(\mathscr{S}_{r}^{r}\right)^{\prime}\left(\mathbb{R}^{d}\right)$.

Proof of Theorem 2. We show the proof of theorem 2 as $r>\frac{1}{2}$. Since $k$ is continuous, the bilinear form $\mathbb{B}$ on $\mathscr{S}_{r, a}^{r, B}\left(\mathbb{R}^{d_{1}}\right) \times \mathscr{S}_{r, a^{\prime}}^{r, B^{\prime}}\left(\mathbb{R}^{d_{2}}\right)$, for any $a, B \in(0, \infty)^{d_{1}}$ and $a^{\prime}, B^{\prime} \in(0, \infty)^{d_{2}}$, $\mathbb{B}(\varphi, \psi)=\langle k \psi, \varphi\rangle, \varphi \in \mathscr{S}_{r, a}^{r, B}\left(\mathbb{R}^{d_{1}}\right), \psi \in \mathscr{S}_{r, a^{\prime}}^{r, B^{\prime}}\left(\mathbb{R}^{d_{2}}\right)$

is separately continuous. Since $\mathscr{S}_{r, a}^{r, B}\left(\mathbb{R}^{d_{1}}\right)$ and $\mathscr{S}_{r, a^{\prime}}^{r, B^{\prime}}\left(\mathbb{R}^{d_{2}}\right)$ is Fréchet space, $\mathbb{B}$ is continuous. Hence we obtain that there exists a constant $C_{a, a^{\prime}, B, B^{\prime}}>0$ such that

$$
|\langle k \psi, \varphi\rangle| \leq C_{a, a^{\prime}, B, B^{\prime}}\|\varphi\|_{\delta \rho}\|\psi\|_{\delta^{\prime} \rho^{\prime}}
$$

Set for $\left(x_{1}, x_{2}\right) \in \mathbb{R}^{d_{1}} \times \mathbb{R}^{d_{2}}$ and $t>0$,

$$
K_{t}\left(x_{1}, x_{2}\right)=\left\langle k E\left(x_{2}-\cdot, t\right), E\left(x_{1}-\cdot, t\right)\right\rangle .
$$


Now we show $K_{t}$ converges in $\left(\mathscr{S}_{r}^{r}\right)^{\prime}\left(\mathbb{R}^{d_{1}} \times \mathbb{R}^{d_{2}}\right)$ as $t \rightarrow 0$. By $(\sharp)$ and Proposition 9 , for any $\varepsilon, \varepsilon^{\prime}>0$, there exists a constant $C_{\varepsilon, \varepsilon^{\prime}}>0$ such that

$$
\left|K_{t}\left(x_{1}, x_{2}\right)\right| \leq C_{\varepsilon, \varepsilon^{\prime}} \exp \left[\varepsilon\left(\left|x_{1}\right|^{\frac{1}{r}}+(1 / t)^{1 /(2 r-1)}\right)\right] \exp \left[\varepsilon^{\prime}\left(\left|x_{2}\right|^{\frac{1}{r}}+(1 / t)^{1 /(2 r-1)}\right)\right] .
$$

Moreover we obtain

$$
\left(\frac{\partial}{\partial t}-\Delta\right) K_{t}\left(x_{1}, x_{2}\right)=0
$$

Therefore, by Proposition 11, there exists $K_{0} \in\left(\mathscr{S}_{r}^{r}\right)^{\prime}\left(\mathbb{R}^{d_{1}} \times \mathbb{R}^{d_{2}}\right)$ such that $K_{0}=\lim _{t \rightarrow 0} K_{t}$ in $\left(\mathscr{S}_{r}^{r}\right)^{\prime}\left(\mathbb{R}^{d_{1}} \times \mathbb{R}^{d_{2}}\right)$.

For $\varphi \in \mathscr{S}_{r}^{r}\left(\mathbb{R}_{x_{1}}^{d}\right), \psi \in \mathscr{S}_{r}^{r}\left(\mathbb{R}_{x_{2}}^{d}\right)$,

$$
\begin{aligned}
\left\langle K_{t}, \varphi \otimes \psi\right\rangle & =\iint_{\mathbb{R}^{d_{1}+d_{2}}} K_{t}\left(x_{1}, x_{2}\right) \varphi\left(x_{1}\right) \psi\left(x_{2}\right) d x_{1} d x_{2} \\
& =\iint_{\mathbb{R}^{d_{1}+d_{2}}}\left\langle k E\left(x_{2}-y_{2}, t\right) \psi\left(x_{2}\right), E\left(x_{1}-y_{1}, t\right) \varphi\left(x_{1}\right)\right\rangle d x_{1} d x_{2} .
\end{aligned}
$$

Since the Riemann sum of an integral converges in $\mathscr{S}_{r}^{r}$, we obtain

$$
\left\langle K_{t}, \varphi \otimes \psi\right\rangle=\left\langle k \int_{\mathbb{R}^{d_{2}}} E\left(x_{2}-y_{2}, t\right) \psi\left(x_{2}\right), \int_{\mathbb{R}^{d_{1}}} E\left(x_{1}-y_{1}, t\right) \varphi\left(x_{1}\right)\right\rangle .
$$

Therefore, by Proposition 10, we obtain

$$
\left\langle K_{0}, \varphi \otimes \psi\right\rangle=\langle k \psi, \varphi\rangle,
$$

as $t \rightarrow 0$.

Similarly, we can also show the proof of Theorem 2 as $r=\frac{1}{2}$.

Remark 4. Z. Lozanov-Crvenković and D. Perišić also proved the Schwartz kernel theorem for the spaces of tempered ultradistributions in [6] by means of the Hermite expansions.

We define the Weyl transform with symbol $T \in\left(\mathscr{S}_{r}^{r}\right)^{\prime}$ by

$$
\langle\mathscr{W}(T) \varphi, \psi\rangle=\langle T, V(\varphi, \bar{\psi})\rangle, \varphi, \psi \in \mathscr{S}_{r}^{r}\left(\mathbb{R}^{d}\right),
$$

where $V(\varphi, \bar{\psi})$ is the Fourier-Wigner transform of $\varphi$ and $\bar{\psi}$. It follows from Proposition 4 that this definition is well defined. M. Cappiello, T. Gramchev and L. Rodino also showed this subject in [1]. We obtain the following result from Theorem 2.

Corollary 3. The map $\mathbb{W}$ from $\mathscr{S}\left(\mathbb{R}^{2 d}\right)$ to the space of bounded operators on $L^{2}\left(\mathbb{R}^{d}\right)$, defined by

$$
\mathscr{W}(F) \varphi(\xi)=\iint_{\mathbb{R}^{2 d}} F(x, y)[\pi(x, y) \varphi](\xi) d x d y, \varphi \in L^{2}\left(\mathbb{R}^{d}\right),
$$

extends uniquely to a bijection from $\left(\mathscr{S}_{r}^{r}\right)^{\prime}\left(\mathbb{R}^{2 d}\right), r \geq 1 / 2$, to the space of continuous linear maps from $\mathscr{S}_{r}^{r}\left(\mathbb{R}^{d}\right), r \geq 1 / 2$, to $\left(\mathscr{S}_{r}^{r}\right)^{\prime}\left(\mathbb{R}^{d}\right), r \geq 1 / 2$. 
Proof. Let $k$ be a continuous linear map from $\mathscr{S}_{r}^{r}\left(\mathbb{R}^{d}\right)$ to $\left(\mathscr{S}_{r}^{r}\right)^{\prime}\left(\mathbb{R}^{d}\right)$. By Theorem 2 , for any $k$, there exists $K \in\left(\mathscr{S}_{r}^{r}\right)^{\prime}\left(\mathbb{R}^{2 d}\right)$ such that

$$
\langle k \varphi, \psi\rangle=\langle K, \varphi \otimes \psi\rangle, \varphi, \psi \in \mathscr{S}_{r}^{r}\left(\mathbb{R}^{d}\right) .
$$

So we have

$$
\begin{aligned}
\langle k \varphi, \psi\rangle & =\langle K, \varphi \otimes \psi\rangle \\
& =\left\langle\mathscr{F}_{1} \mathbf{S} K, V(\varphi, \bar{\psi})\right\rangle,
\end{aligned}
$$

where $\mathscr{F}_{1}$ is the Fourier transform of the first variable and $\mathbf{S}$ is defined by $\mathbf{S} h(a, b)=h\left(a+\frac{b}{2}\right.$, $\left.a-\frac{b}{2}\right)$. Set $T=\mathscr{F}_{1} \mathbf{S} K$,

$$
\begin{aligned}
(4.1) & =\langle T, V(\varphi, \bar{\psi})\rangle \\
& =\langle\mathscr{W}(T) \varphi, \psi\rangle .
\end{aligned}
$$

Since $\mathscr{F}_{1} \mathbf{S} K \in\left(\mathscr{S}_{r}^{r}\right)^{\prime}\left(\mathbb{R}^{2 d}\right)$, for any $k$, there exists $T \in\left(\mathscr{S}_{r}^{r}\right)^{\prime}\left(\mathbb{R}^{2 d}\right)$ such that $k=\mathscr{W}(T)$.

Remark 5. Z. Lozanov-Crvenković and D. Perišić gave the similar result for $\left(\mathscr{S}_{r}^{r}\right)^{\prime}$ as $r>1$ in [6].

\section{References}

[1] Cappiello, M., Gramchev, M.T. and Rodino, L., Gelfand-Shilov spaces, pseudodifferential operators and localization operators, in Modern Trends in PseudoDifferential Operators, Editors: Toft, J., Wong, M.W. and Zhu, H., Birkhäuser, 297-312.

[2] Chung, S.-Y., Kim, D. AND LeE, E.G., Schwartz kernel theorem for the Fourier hyperfunctions, Tsukuba J. Math., Vol. 19, N.2 (1995), 377-385.

[3] Dong, C. And Matsuzawa, T., $\mathscr{S}$-space of Gel'fand-Shilov and differential equations, Japan. J. Math. Vol. 19, N.2, (1994), 227-239.

[4] Gel'Fand, I.M. AND Shilov, G.E., Generalized Functions Vol. 2, Academy of Sciences Moscow, U.S.S.R, 1958

[5] Gröchenig, K. And Zimmermann, G., Spaces of test functions via the STFT, J. Function Spaces Appl., 2 (2004), 25-53.

[6] Lozanov-Crvenković, Z. AND PerišIć, D., Kernel theorem for the space of tempered ultradistributions, Integral Transforms and Special Functions, Vol. 18, N.10, October (2007), 699-713. 
[7] Nagamachi, S. AND Mugibayashi, N., Hyperfunction quantum field theory, Commun. math. Phys., 46 (1976), 119-134.

[8] OKA, Y., $N$-representation for $\mathscr{S}$ and $\mathscr{S}^{\prime}$, Sophia Univ. Master's Thesis, 2002.

[9] PooL, J.C.T., Mathematical aspects of the Weyl correspondence, J. Math. Phys. vol. 7, N.1, January (1966), 66-76.

[10] Simon, B., Distributions and their Hermite expansions, J. Math. Phys. vol. 12, N.1 (1971), 140-148.

[11] Simon, B., The Weyl transform and $L^{p}$ functions on phase space, Proc. Amer. Math. Soc., 116 (1992), 1045-1047.

[12] Tofт, J., Continuity properties for modulation spaces with applications to pseudodifferential calculus, I, J. Funct. Anal., 207 (2), (2004), 399-429.

[13] Voros, A., An algebra of pseudodifferential operators and the asymptotics of quantum mechanics, J. Funct. Anal., 29 (1978), 104-132.

[14] WeYL, H., The Theory of Groups and Quantum Mechanics, Dover, New York, 1950.

[15] Wong, M.W., Weyl Transforms, Springer-Verlag, New York, Inc., 1998.

[16] Yoshino, K. AND OKA, Y., Asymptotic expansions of the solutions to the heat equations with hyperfunctions initial value, Commun. Korean Math. Soc., 23 (2008), N.4, 555-565.

[17] Zhang, G.-Z., Theory of distributions of $S$ type and pansions, Chinese Math. Acta., 4 (1963), 211-221. 